\title{
Molecular Imaging of Post-Src Inhibition Tumor Signatures for Guiding Dasatinib Combination Therapy
}

\author{
Liquan Gao*1, Hao Liu*1, Xianlei Sun ${ }^{1}$, Duo Gao ${ }^{1}$, Chenran Zhang ${ }^{1}$, Bing Jia ${ }^{1}$, Zhaohui Zhu², Fan Wang ${ }^{1,3}$, \\ and Zhaofei Liu ${ }^{1}$ \\ ${ }^{I}$ Medical Isotopes Research Center and Department of Radiation Medicine, School of Basic Medical Sciences, Peking University \\ Health Science Center, Beijing, China; ${ }^{2}$ Department of Nuclear Medicine, Peking Union Medical College Hospital, Beijing, China; \\ and ${ }^{3}$ Interdisciplinary Laboratory, Institute of Biophysics, Chinese Academy of Sciences, Beijing, China
}

Noninvasive, real-time, quantitative measurement of key biomarkers associated with cancer therapeutic interventions could provide a better understanding of cancer biology. We investigated in this study whether incorporating multiple molecular imaging approaches could be used to guide dasatinib anti-Src therapy and aid in the rational design of a combination therapy regimen. Methods: Bioluminescence imaging, ${ }^{18} \mathrm{~F}$-FDG PET, integrin $\alpha_{v} \beta_{3}$-targeted SPECT/ $\mathrm{CT}$, and vascular endothelial growth factor-targeted near-infrared fluorescence imaging were performed before and after dasatinib treatment in a tumor mouse model. Results: There was no significant difference in the bioluminescence imaging signal or ${ }^{18} \mathrm{~F}-\mathrm{FDG}$ tumor uptake in dasatinib-treated tumors compared with the control tumors. However, the uptake of 99mT-3PRGD2 (integrin $a_{v} \beta_{3}-$ specific) and DyLight755-ranibizumab (vascular endothelial growth factor-specific) in the dasatinib-treated tumors was significantly lower than that in the control tumors. In vitro studies confirmed the antiangiogenic effects of dasatinib but indicated a lack of cytotoxicity. Dasatinib plus cytotoxic docetaxel elicited marked synergistic tumor growth inhibition in vivo. Conclusion: Visualization of post-Src inhibition tumor signatures through multiple imaging approaches facilitates sensitive and quantitative measurement of cancer biomarkers in vivo, thus aiding in the rational design of dasatinib combination therapy.

Key Words: image-guided therapy; angiogenesis; tumor response; dasatinib; Src family of kinases

J Nucl Med 2016; 57:321-326

DOI: 10.2967/jnumed.115.158881

$\mathbf{T}$ he Src family kinases (SFKs) are a large family of nonreceptor protein tyrosine kinases, which regulate numerous intracellular signaling pathways responsible for tumor cell proliferation, invasion, angiogenesis, and metastasis (1). SFK activity is upregulated in many malignant tumor types, thus representing a promising target for tumor therapy (2). Dasatinib (BMS-354825) is an orally bioavailable small-molecule Src inhibitor that has been approved for

\footnotetext{
Received Apr. 6, 2015; revision accepted Aug. 26, 2015.

For correspondence contact: Zhaofei Liu, Medical Isotopes Research Center, School of Basic Medical Sciences, Peking University, Beijing 100191, China.

E-mail: liuzf@bjmu.edu.cn

*Contributed equally to this work.

Published online Sep. 17, 2015.

COPYRIGHT (C 2016 by the Society of Nuclear Medicine and Molecular Imaging, Inc.
}

chronic myelogenous leukemia and is being extensively studied in both preclinical and clinical studies as a promising agent for the management of various solid tumors $(1,2)$.

Despite significant progresses in dasatinib-based Src-targeted therapy, many tumors relapse after an initial response and become refractory to therapy, consequently leading to treatment failure. To overcome dasatinib resistance, numerous combination therapeutic regimens have been evaluated. Although promising in some cases, several studies have demonstrated the failure of dasatinib combination therapy or reported controversial outcomes. One study in 2 patients with castration-resistant prostate cancer showed that dasatinib plus docetaxel (DTX) provided meaningful clinical benefits and delayed disease progression (3). However, in a randomized, doubleblind phase 3 trial in patients with castration-resistant prostate cancer, dasatinib plus DTX was not found to improve overall survival or to improve any measures of patient benefit (4); these results highlight the importance of a rational design of a combination therapy regimen toward personalized cancer treatment.

A critical step in personalized cancer therapy is the development of effective noninvasive in vivo imaging techniques for predicting or monitoring tumor response at the earliest stages. However, single-modality imaging is not sufficient for assessing the multiple molecular signatures of some cancers. Integration of various noninvasive imaging modalities gives a readout of multiple cancer biomarkers, thus providing complementary information at a functional and anatomic level (5).

Several molecular imaging probes have been investigated for noninvasively monitoring tumor responses to therapies. For example, ${ }^{18} \mathrm{~F}-\mathrm{FDG}$ PET is commonly used in the clinical setting for visualization of tumor glucose metabolism in vivo (6). Integrin $\alpha_{v} \beta_{3}$ is overexpressed in numerous tumor cells and activated endothelial cells during tumor angiogenesis. SPECT imaging using ${ }^{99 \mathrm{~m} T c-3 P R G D 2}$ (7), a ${ }^{99 \mathrm{~m}} \mathrm{Tc}-$ labeled arginine-glycine-aspartic acid (RGD)-based radiotracer, has been used to visualize and quantify tumor integrin $\alpha_{v} \beta_{3}$ expression in vivo. Further, vascular endothelial growth factor (VEGF) is a key inducer of tumor angiogenesis. Recent studies have demonstrated that near-infrared fluorescence (NIRF) dye-labeled anti-VEGF antibodies can specifically detect changes in VEGF in response to cancer therapies $(8-10)$.

In this study, to visualize several different tumor signatures with high contrast and specificity, we performed bioluminescence imaging (BLI), ${ }^{18}$ F-FDG PET, integrin $\alpha_{v} \beta_{3}$ SPECT, and VEGF-targeted NIRF imaging, simultaneously, in a human lung cancer mouse model treated with dasatinib. Noninvasive multiparameter imaging enabled the in vivo detection of early tumor changes during dasatinib therapy, 
and the data obtained facilitated the rational design of a more efficacious strategy for dasatinib combination therapy.

\section{MATERIALS AND METHODS}

\section{Cell Culture and Animal Model}

The A549 human non-small cell lung cancer cell line was obtained from American Type Culture Collection. Firefly luciferase stably transfected A549 (A549-fLuc) cells were generated using a previously described method (11). A robust linear correlation between the A549fLuc cell number and fLuc activity was observed $\left(R^{2}=0.9915, P<\right.$ 0.0001; Supplemental Figs. 1A and 1B [supplemental materials are available at http://jnm.snmjournals.org]), and no significant difference between A549 and A549-fLuc cells was observed in terms of proliferation (Supplemental Fig. 1C).

All animal experiments were performed in accordance with the Guidelines of Peking University Animal Care and Use Committee. The A549-fLuc subcutaneous tumor model was established as described in the supplemental materials and methods.

\section{In Vivo Dasatinib Treatment}

A549-fLuc tumor-bearing BALB/c nude mice with a tumor size of 150-200 $\mathrm{mm}^{3}$ were segregated into 6 groups: BLI/NIRF imaging control $(n=7)$, BLI/NIRF imaging treatment $(n=7)$, PET control $(n=5)$, PET treatment $(n=5)$, SPECT control $(n=5)$, and SPECT treatment $(n=5)$. A maximum-tolerated dose of $80 \mathrm{mg} / \mathrm{kg} / \mathrm{d}$ for dasatinib ( $6 \mathrm{~d}$ of continuous administration) was determined in our pilot study, and $50 \%$ of the maximum tolerated dose was used in this study. After the baseline imaging (day 0), animals in the treatment groups were administered dasatinib (40 $\mathrm{mg} / \mathrm{kg} / \mathrm{d}$ ) (in 1:1 propylene glycol/water) via gavage for $6 \mathrm{~d}$ (days 1-6). Animals in the control groups were administered 1:1 propylene glycol/water (vehicle control) using the same protocol. Molecular imaging was repeated on day 7 (the schedule of dasatinib treatment and imaging are shown in Fig. 1A). Immediately after the imaging studies, animals in the PET control and treatment and SPECT control and treatment groups were sacrificed. The tumors were harvested, immediately frozen in optimalcutting-temperature medium, and then cut into 5- $\mu$ m-thick slices for immunofluorescence staining. The tumor size in the BLI/NIRF control and BLI/NIRF treatment groups was measured every $4 \mathrm{~d}$ to observe the effect of dasatinib on the tumor growth.

\section{BLI}

Detailed procedures of BLI are described in the supplemental materials and methods. The BLI signal intensity in the tumor regions was quantified as the sum of all detected photon counts within the region of interest after subtraction of background luminescence.

\section{${ }^{18}$ F-FDG PET}

${ }^{18} \mathrm{~F}-\mathrm{FDG}$ PET scans and image analysis were performed using a microPET R4 rodent model scanner (Siemens Medical Solutions) as previously described $(12,13)$. Each A549-fLuc tumor-bearing mouse was intravenously injected with $3.7 \mathrm{MBq}$ of ${ }^{18} \mathrm{~F}-\mathrm{FDG}$, and 5-min static PET scans were acquired at $1 \mathrm{~h}$ after injection. Images were reconstructed, and the region-of-interest-derived tumor percentage injected dose per gram was determined.

\section{9mTC-3PRGD2 SPECT/CT}

The integrin $\alpha_{\mathrm{v}} \beta_{3}$-targeting SPECT radiotracer ${ }^{99 \mathrm{~m} T c-3 P R G D 2}$ was prepared as previously described (7). Each A549-fLuc tumorbearing nude mouse was intravenously administered $18.5 \mathrm{MBq}$ of ${ }^{99 \mathrm{~m} T \mathrm{Tc}-3 P R G D 2 .}$ SPECT and helical CT scans of the mice were obtained at $1 \mathrm{~h}$ after injection using a small-animal NanoScan SPECT/CT imaging system (Mediso). The region of interest encompassed the tumor and muscle (background), and the tumor-to-muscle radioactivity (counts $/ \mathrm{mm}^{3}$ ) ratio was calculated.

\section{DyLight755-Ranibizumab (Dye755-Ran) NIRF}

The human VEGF-specific NIRF imaging agent Dye755-Ran was prepared as previously described (9). Each A549-fLuc tumorbearing nude mouse was intravenously injected with $0.5 \mathrm{nmol}$ of Dye755-Ran. In vivo small-animal NIRF imaging was then performed at $4 \mathrm{~h}$ after injection using the IVIS spectrum system (Xenogen). The region of interest was highlighted for each tumor, and the fluorescence intensity was presented as the average radiant efficiency in the unit of $\left(\mathrm{p} / \mathrm{s} / \mathrm{cm}^{2} / \mathrm{sr}\right) /\left(\mu \mathrm{W} / \mathrm{cm}^{2}\right)$.

\section{Immunofluorescence Staining and Analyses}

A549-fLuc tumor tissues were stained and analyzed for glucose transporter 1 (GLUT-1), Ki-67 proliferation marker, human integrin $\alpha_{v} \beta_{3}$, murine integrin $\beta 3$, and human VEGF. Detailed procedures are described in the supplemental materials and methods.

\section{Radioligand Binding Assay}

The effect of dasatinib on integrin $\alpha_{\mathrm{v}} \beta_{3}$ inactivation was determined using cell radioligand binding assays. Detailed procedures are described in the supplemental materials and methods.

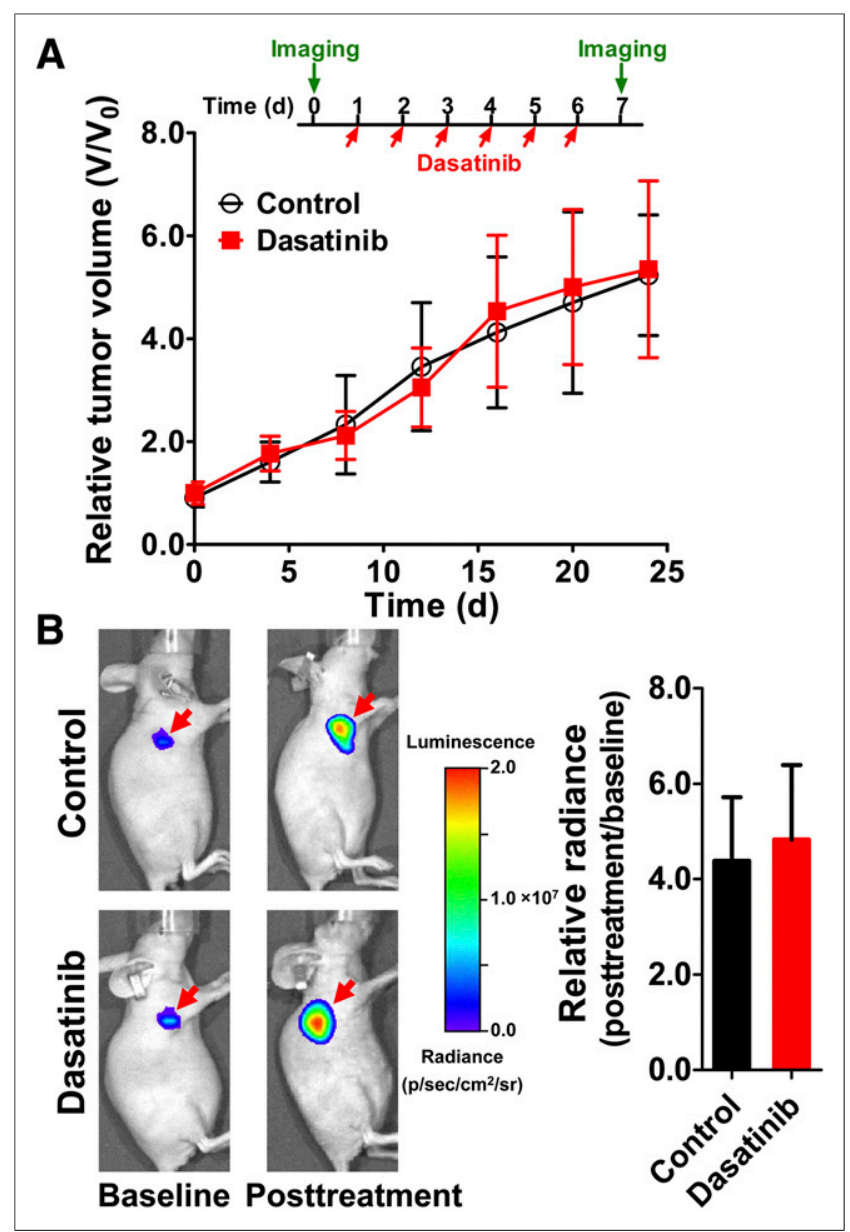

FIGURE 1. Dasatinib had no effect on A549-fLuc tumor growth inhibition. (A) Tumor growth curves of A549-fLuc tumor-bearing mice administered vehicle control or dasatinib daily for $6 \mathrm{~d}$ ( $n=7 /$ group). Inset, schedule of dasatinib treatment and molecular imaging experiments. (B) Representative bioluminescence images and tumor BLI signal intensity changes from baseline to after treatment in the A549fLuc tumor-bearing mice ( $n=7 /$ group). Arrows indicate location of tumors. 


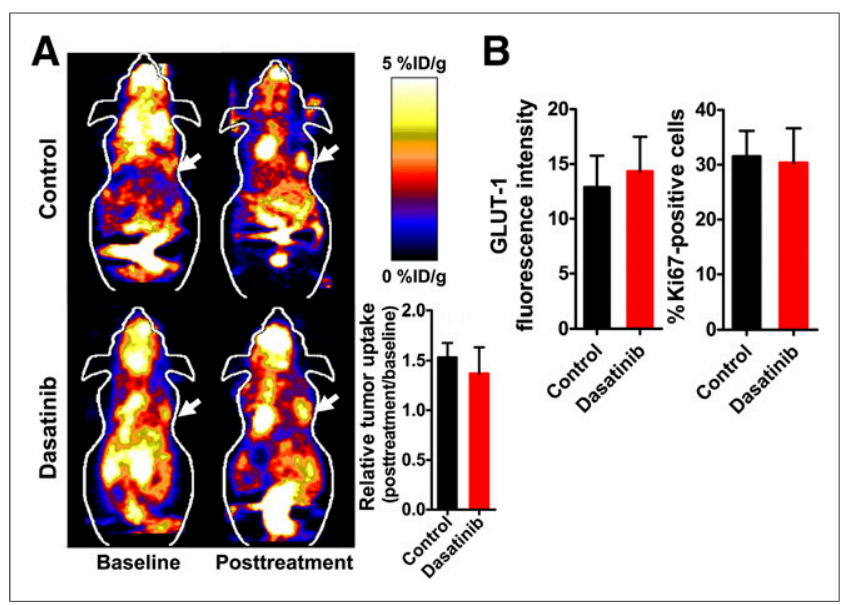

FIGURE 2. ${ }^{18} \mathrm{~F}-\mathrm{FDG}$ PET imaging revealed comparable tumor cell metabolism in A549-fLuc tumors with or without dasatinib treatment. (A) Representative small-animal PET images and relative tumor ${ }^{18} \mathrm{~F}-\mathrm{FDG}$ uptake (posttreatment/baseline ratios) in A549-fLuc tumor-bearing mice ( $n=5$ /group). Arrows indicate location of tumors. (B) Quantified GLUT-1 fluorescence intensity and quantified percentage of Ki-67-positive cells of the A549-fLuc tumor tissues with or without dasatinib treatment.

\section{Matrigel (BD Biosciences) Plug Assay}

The antiangiogenic effect of dasatinib was evaluated by Matrigel plug assay. Detailed procedures are described in the supplemental materials and methods.

\section{In Vivo Combination Therapy}

Groups ( $n=10 /$ group) of A549-fLuc tumor-bearing BALB/c nude mice with $150-200 \mathrm{~mm}^{3}$ tumors were administered dasatinib $(40 \mathrm{mg} / \mathrm{kg})$ (in 1:1 propylene glycol/water) daily via gavage for $6 \mathrm{~d}$ (days $1-6$ ), with or without intraperitoneal injection of DTX $(5 \mathrm{mg} / \mathrm{kg}$ ) (in $13 \%$ ethanol) on days 1, 3, and 5. Animals in the control group $(n=10)$ were administered 1:1 propylene glycol/water via gavage and injected intraperitoneally with $13 \%$ ethanol. On day 7, 5 animals from each

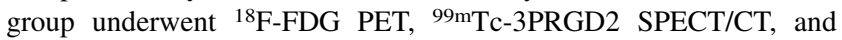
Dye755-Ran NIRF imaging using the same procedures as described above. Immediately after the imaging experiments, 3 animals from each group were sacrificed and the tumor tissues were frozen, sectioned, and stained for $\mathrm{Ki}-67$ to determine the tumor cell proliferation.

\section{Statistical Analysis}

Quantitative data are expressed as mean \pm SD. Means were compared using the Student $t$ test. $P$ values of less than 0.05 were considered statistically significant.

\section{RESULTS}

\section{Dasatinib Had No Antitumor Effects in A549-fLuc Tumor Model}

As shown in Figure 1A, the tumor growth curve in the dasatinib treatment group was almost identical to that in the control group, suggesting that the antitumor effect of dasatinib was negligible.

\section{BLI and PET Revealed That Dasatinib Had No Effect on Tumor Cell Proliferation}

As shown in Figure 1B, there was a significant increase in BLI signal intensity in both control and dasatinib treatment groups as tumors grew between days 0 and 7. The relative tumor BLI signal intensities (posttreatment/baseline ratio) were $4.38 \pm 1.33$ and $4.83 \pm 1.56(P>0.05)$ for the control and dasatinib treatment groups, respectively. These results demonstrated that $6 \mathrm{~d}$ of dasatinib treatment did not lead to tumor BLI signal reduction in this model.

Similar to the results of BLI, the ${ }^{18}$ F-FDG tumor uptake on day 7 was significantly increased in both the control and the dasatinib treatment groups compared with the baseline, as a result of tumor growth. There was no significant change in the relative ${ }^{18} \mathrm{~F}-\mathrm{FDG}$ tumor uptake (posttreatment/baseline ratio) between the control and treatment groups $(1.53 \pm 0.14$ vs. $1.37 \pm 0.26 ; P>0.05$; Fig. 2A). To validate the in vivo PET imaging observations on tumor cell proliferation, the tumor tissues were stained for GLUT-1 and Ki-67, markers reflecting tumor glucose metabolism and cell DNA synthesis, respectively. We observed that tumors in both the control and the dasatinib treatment groups showed high expression of GLUT-1 and moderate expression of Ki-67 (Supplemental Figs. $2 \mathrm{~A}$ and $2 \mathrm{~B}$ ). There were no statistical differences in the GLUT-1 and Ki-67 expressions in the A549-fLuc tumors followed by the dasatinib treatment (Fig. 2B).

\section{SPECT/CT Revealed That Dasatinib Showed Antiangiogenic Effects and Could Inactivate Integrin $\boldsymbol{\alpha}_{\mathrm{v}} \boldsymbol{\beta}_{3}$}

To investigate whether dasatinib had any effects on other tumor signatures such as tumor angiogenesis, we performed smallanimal SPECT/CT using an integrin $\alpha_{\mathrm{v}} \beta_{3}$-specific radiotracer,

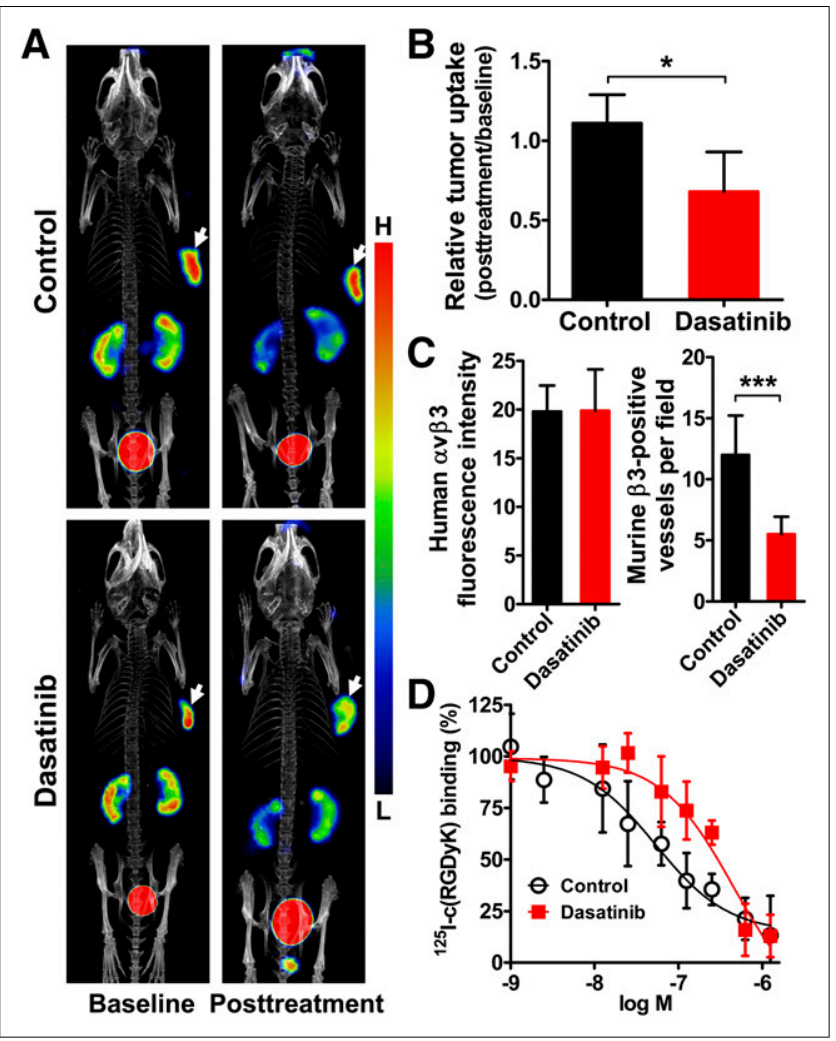

FIGURE 3. ${ }^{99 m T C-3 P R G D 2 ~ S P E C T / C T ~ i m a g i n g ~ r e v e a l e d ~ d e c r e a s e d ~}$ murine integrin $\beta 3$ expression and inactivated integrin $\alpha_{v} \beta_{3}$ after dasatinib treatment. ( $A$ and $B$ ) Representative small-animal SPECT/CT images (A) and relative tumor $99 \mathrm{mTC}-3 P R G D 2$ uptake (posttreatment/ baseline ratios; B) in A549-fLuc tumor-bearing mice ( $n=5 /$ group). Arrows indicate location of tumors. (C) Quantified human integrin $a_{v} \beta_{3}$ fluorescence intensity and quantified density of murine integrin $\beta 3-$ positive vessels in dasatinib-treated and control A549-fLuc tumor tissues. (D) ${ }^{125} \mathrm{I}-\mathrm{C}(\mathrm{RGDyK})$ radioligand binding assay in dasatinib-treated and control A549-fLuc tumor cells. ${ }^{*} P<0.05 .{ }^{* \star} P<0.001$. 


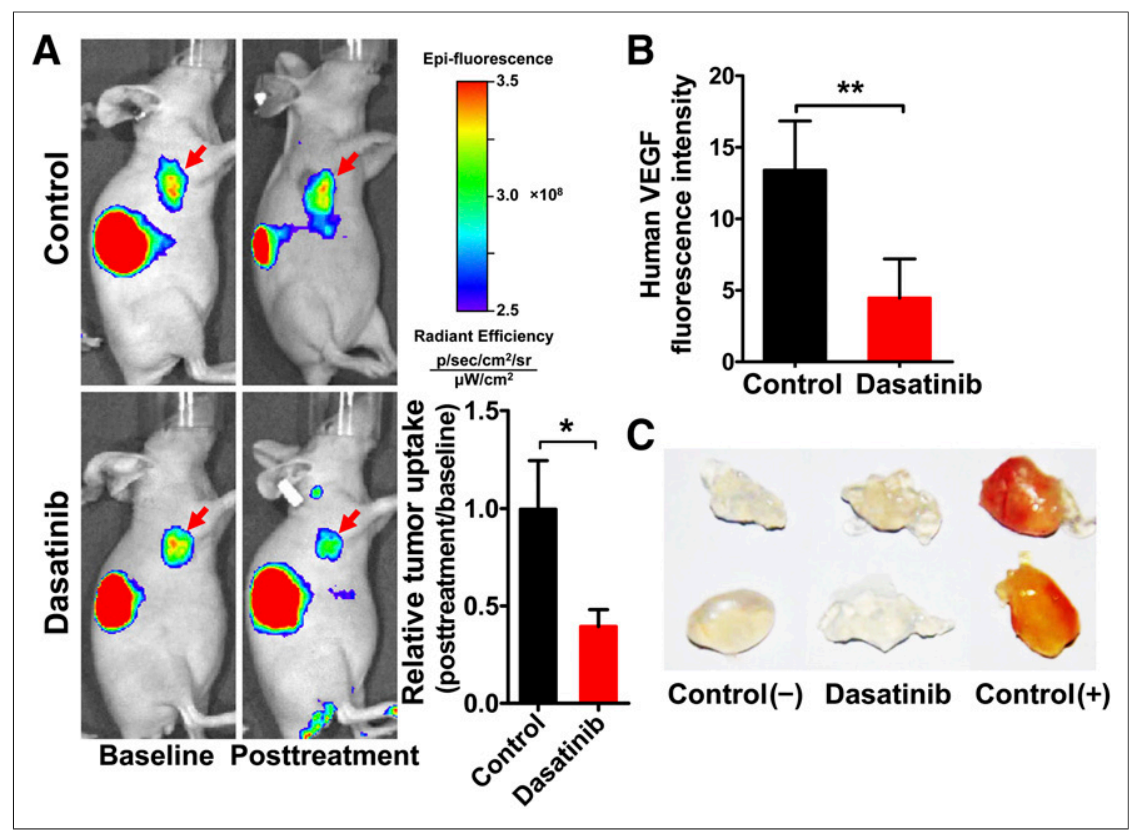

FIGURE 4. NIRF imaging using Dye755-Ran revealed antiangiogenic effects of dasatinib. (A) Representative NIRF images and relative tumor Dye755-Ran uptake (posttreatment/baseline ratios) in A549-fLuc tumor-bearing mice ( $n=7 /$ group). Arrows indicate location of tumors. (B) Quantified human VEGF fluorescence intensity in dasatinib-treated and control A549-fLuc tumor tissues. (C) Representative Matrigel plugs harvested from nude mice after $7 \mathrm{~d}$ of inoculation. Control $(-)=$ Matrigel only; control $(+)=$ Matrigel mixed with dimethyl sulfoxide (vehicle control) and growth factors; dasatinib $=$ Matrigel mixed with dasatinib and growth factors. ${ }^{\star} P<0.05$. ${ }^{\star *} P<0.01$.
Dye755-Ran. As shown in Figure 4A, the tumor uptake of Dye755-Ran was significantly decreased on dasatinib treatment. The relative Dye755-Ran tumor uptake (posttreatment/baseline ratio) in the treatment group was significantly lower than that in the control group $(0.39 \pm 0.09$ vs. $0.99 \pm 0.25 ; P<0.05)$. Ex vivo tumor tissue immunofluorescence imaging validated that the VEGF expression in the dasatinibtreated tumors was significantly lower than that in the control tumors $(4.46 \pm$ 2.74 vs. $13.40 \pm 3.45 ; P<0.01$; Fig. $4 \mathrm{~B}$; Supplemental Fig. 4).

We next performed a Matrigel plug assay to determine the antiangiogenic effects of dasatinib in vivo. As shown in Figure 4C, dasatinib showed antitumor angiogenesis effects compared with the vehicle group (control $[+]$ ). Without growth factors, Matrigel alone did not induce host blood vessel formation in the mice (control $[-]$ ).

\section{Dasatinib Inhibited Tumor Cell \\ Migration But Had Limited \\ Cellular Toxicity}

We observed that dasatinib inhibited the migration of A549-fLuc cells in a dosedependent manner (Supplemental Figs. 5A ${ }^{99 m}$ Tc-3PRGD2. The relative ${ }^{99 m}$ Tc-3PRGD2 tumor uptake (posttreatment/baseline ratio) in the treatment group was significantly lower than that in the control group $(0.68 \pm 0.25$ vs. $1.11 \pm 0.18$; $P<0.05$; Figs. 3A and 3B).

In a mouse model bearing human tumor xenografts such as was used in the present study, the integrin $\alpha_{v} \beta_{3}$ expression on tumor vascular endothelial cells is of murine origin, whereas that on the tumor cells is of human origin (14). Moreover, radiolabeled RGD peptides could recognize both human and murine integrin $\alpha_{\mathrm{v}} \beta_{3}$ (14). Therefore, we stained the tumor sections with both antihuman integrin $\alpha_{\mathrm{v}} \beta_{3}$ and antimurine integrin $\beta 3$ antibodies to validate the tumor targeting of ${ }^{99 \mathrm{~m}} \mathrm{Tc}-3 \mathrm{PRGD} 2$. As shown in Figure $3 \mathrm{C}$ and Supplemental Figure 3A, A549-fLuc tumor cells exhibited positive human integrin $\alpha_{\mathrm{v}} \beta_{3}$ expression, which was not affected by dasatinib treatment. In contrast, the murine integrin $\beta 3$ expression levels in the A549-fLuc tumor tissues were significantly decreased after dasatinib treatment $(P<0.001$; Fig. 3C; Supplemental Fig. 3B).

Src inhibitors such as dasatinib have been demonstrated to have effects on integrin $\alpha_{\mathrm{v}} \beta_{3}$ inactivation, and inactivated integrin $\alpha_{\mathrm{v}} \beta_{3}$ has been shown to have low affinity for RGD peptides $(15,16)$. We thus performed radioligand binding assays to determine the receptor binding affinity of RGD for A549-fLuc cells with or without dasatinib treatment. As shown in Figure 3D, the best-fit 50\% inhibitory concentration values were $(45.56 \pm 4.31) \times 10^{-8} \mathrm{M}$ and $(5.38 \pm$ 1.63) $\times 10^{-8} \mathrm{M}$ for the dasatinib-treated tumor cells and untreated cells, respectively. These results demonstrated that dasatinib treatment significantly reduced RGD binding affinity for integrin $\alpha_{v} \beta_{3}$.

\section{NIRF Imaging Confirmed Antiangiogenic Effects of Dasatinib}

To confirm the antiangiogenic effects of dasatinib, we performed NIRF imaging using a VEGF-specific fluorescent agent, and 5B). However, our cell viability studies showed that dasatinib had only a minor effect on cell toxicity. At a high concentration of $1 \mu \mathrm{M}$, greater than $80 \%$ cell viability was observed (Supplemental Fig. 5C).

\section{Improved Antitumor Effects of Combination Therapy Using Antiangiogenic Dasatinib and Cytotoxic DTX}

In contrast to the results observed in the dasatinib treatment group, $10 \mathrm{nM}$ DTX exhibited evident cytotoxicity; the cell viability was less than 50\% (Supplemental Fig. 5C). In combination with $30 \mathrm{nM}$ dasatinib, the cytotoxic effect of $10 \mathrm{nM}$ DTX was further increased (Supplemental Fig. 5C). We also performed a cell colony formation assay to confirm the cytotoxicity of DTX. The numbers of cell colonies in the DTX and dasatinib plus DTX groups were significantly fewer than those in the control and dasatinib groups (Supplemental Figs. 5D and 5E).

We next assessed the combination of antiangiogenic dasatinib and tumor-cell-toxic DTX in vivo. As shown in Figure 5A, lowdose DTX $(5 \mathrm{mg} / \mathrm{kg})$ had a minor effect on tumor growth inhibition, and the combination of dasatinib plus DTX significantly improved this antitumor effect; the tumor size in the dasatinib plus DTX group was significantly smaller than that in the other groups from day 16 up to the end of this study (day 24). Ex vivo Ki-67 staining confirmed that the tumor cell proliferation index $(\% \mathrm{Ki}-$ 67-positive cells) in the dasatinib plus DTX group (10.26 \pm 3.88$)$ was significantly lower than that in the control $(37.02 \pm 6.26)$, dasatinib $(36.18 \pm 7.89)$, and DTX $(21.46 \pm 2.89)$ groups $(P<$ 0.001; Fig. 5B).

Lastly, noninvasive PET, SPECT/CT, and NIRF imaging studies were performed to determine the tumor signatures after dasatinib plus DTX therapy. ${ }^{18}$ F-FDG PET, ${ }^{99}$ Tc-3PRGD2, and Dye755Ran NIRF imaging all demonstrated significantly reduced tumor signals (Fig. 5C; Supplemental Fig. 6). 


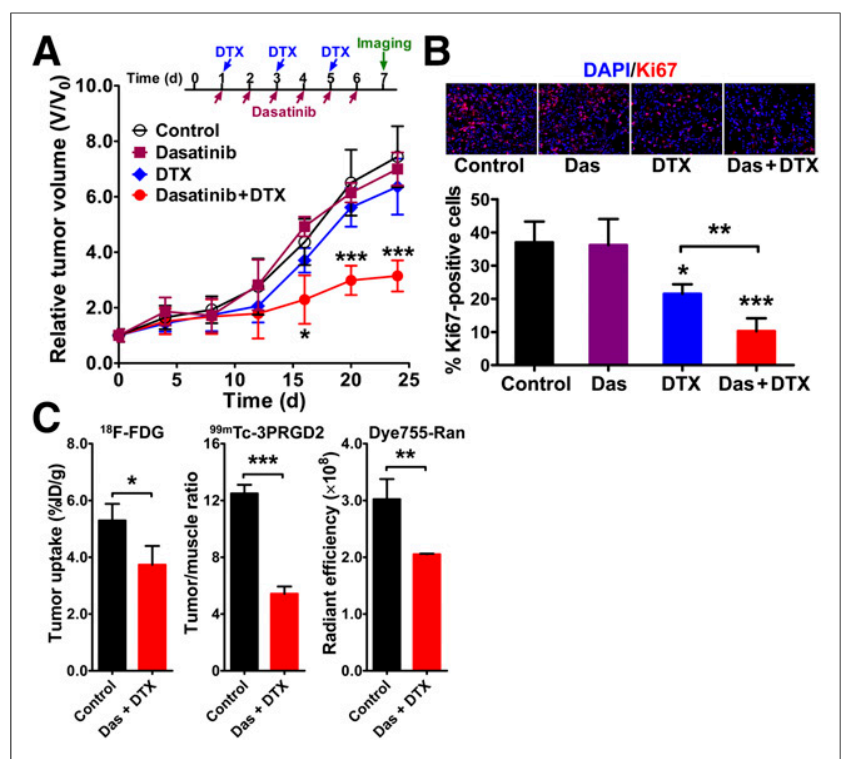

FIGURE 5. Significant improvements were observed in in vivo antitumor effects when combining dasatinib and DTX. (A) Tumor growth curves of A549-fLuc tumor-bearing mice that received daily administration of vehicle control, dasatinib, DTX, or dasatinib plus DTX (Das + DTX) for $6 \mathrm{~d}$ ( $n=7 /$ group). Inset, schedule of combination treatment and molecular imaging experiments. (B) Immunofluorescence staining of $\mathrm{Ki}-67$ and quantified percentage of Ki-67-positive cells in A549-fLuc tumor tissues harvested from mice treated with vehicle (control), dasatinib (Das), DTX, and dasatinib plus DTX (Das + DTX) daily for 6 d. (C) Quantified tumor uptake (percentage injected dose per gram) of ${ }^{18} \mathrm{~F}-\mathrm{FDG}$, tumor-to-muscle ratios of 99mTc-3PRGD2, and tumor fluorescence intensity (radiance efficacy) of Dye755-Ran in A549-fLuc tumor-bearing nude mice with or without dasatinib plus docetaxel (Das + DTX) treatment for $6 \mathrm{~d}$ ( $n=5$ /group). ${ }^{\star} P<0.05 .{ }^{\star \star} P<0.01 .{ }^{* \star \star} P<0.001$.

\section{DISCUSSION}

Cancer therapeutic interventions can lead to multiple changes in the balance and regulation of various signaling pathways, consequently resulting in the upregulation or downregulation of several cancer biologic markers $(17,18)$. Using molecular imaging of different biomarkers, we successfully demonstrated the antiangiogenic effects, but limited cytotoxic effect, of dasatinib in A549-fLuc tumors. The information acquired from the imaging studies successfully guided us to conduct combination therapy by simultaneously targeting the tumor cells (using the cytotoxic drug DTX) along with the tumor vasculature (using antiangiogenic dasatinib). This logical regimen led to synergistic efficacy and significantly improved antitumor effect in the A549-fLuc mouse model.

Similar to in vivo fluorescence imaging, BLI has the inherent limitation of limited tissue penetration depth. In addition, bioluminescence images are planar and thus may affect data reconstruction and quantification (19). In contrast to optical imaging, PET and SPECT are highly sensitive and quantitative and can detect the therapeutic response earlier than anatomic imaging techniques such as CT. Previous studies $(20,21)$ have demonstrated that ${ }^{18} \mathrm{~F}$-FDG imaging has limitations or is not optimal for monitoring therapeutic tumor response, with one of the major reasons for this finding being that ${ }^{18} \mathrm{~F}-\mathrm{FDG}$ is not tumor-specific, as it also shows high uptake in benign conditions such as inflammation. Many solid tumors are perfused with inflammatory cells $(22,23)$, and thus monitoring tumor therapy using ${ }^{18}$ F-FDG may be confused by treatment-induced inflammatory changes $(20,21)$. A previous study demonstrated that dasatinib could inhibit the production of proinflammatory cytokines in vitro (24). However, in this study, dasatinib treatment did not alter the tumor F4/80 levels, a macrophagespecific marker of inflammation (data not shown). Furthermore, both GLUT-1 and Ki-67 staining confirmed that dasatinib treatment did not cause significant changes in tumor glucose metabolic activity or cell proliferation.

Integrin $\alpha_{\mathrm{v}} \beta_{3}$-specific RGD radiotracers have been used to monitor tumor responses to several therapeutic drugs and are known to be superior to ${ }^{18} \mathrm{~F}$-FDG for tumor treatment monitoring (21). In most cases, the rationale for RGD radiotracer-based treatment monitoring is that therapies, especially antiangiogenic drugs, can inhibit tumor angiogenesis by downregulating integrin $\alpha_{\mathrm{v}} \beta_{3}$ levels, although the detailed mechanism regarding this signaling pathway is not clearly understood. Here we confirmed that dasatinib treatment resulted in downregulation of the host (murine) integrin $\beta 3$ (expressed on neovasculature). These results, together with the decreased VEGF expression (Fig. 4B) after dasatinib treatment and the reduced blood vessel formation (Fig. 4C) after dasatinib treatment, confirmed the antiangiogenic effects of dasatinib in vivo.

Src family kinases play a critical role for integrin signaling and activation (25), and thus SFK inhibitors (e.g., dasatinib) may cause integrin inactivation. In terms of integrin $\alpha_{v} \beta_{3}$, its binding affinity to endogenous ligands (e.g., vitronectin, fibronectin, and fibrinogen) or synthesized RGD peptides in the inactive state is much lower than that in the activated state (26). Dumont et al. (16) demonstrated that small-animal PET imaging using ${ }^{64} \mathrm{Cu}$-radiolabeled RGD peptides can specifically detect dasatinib-induced integrin $\alpha_{\mathrm{v}} \beta_{3}$ inactivation, thereby allowing monitoring of the tumor responses to dasatinib therapy before any observed changes in tumor size. Imaging using a radiolabeled RGD peptide reflects the tumor response in the dasatinib-responsive U87MG animal model (16); however, in the dasatinib-resistant (Fig. 1A) A549-fLuc tumor model, Src inhibition using dasatinib treatment also led to a reduction in the tumor uptake of the radiolabeled RGD peptide (Figs. 3A and 3B). These results suggest that PET or SPECT using radiolabeled RGD peptides is not sufficient to identify whether the tumor is responsive or resistant to dasatinib therapy, which further highlights the merits of multiple tumor signature imaging.

We observed a significantly reduced RGD binding affinity for integrin $\alpha_{v} \beta_{3}$ in the dasatinib-treated tumor cells (Fig. 3D). These results, together with the remarkable effect of dasatinib on the downregulation of murine integrin $\beta 3$ (Fig. 3C), may be the main reason for the significantly reduced uptake of ${ }^{99 m}$ Tc-3PRGD2 in the dasatinib-treated tumors (Figs. 3A and 3B). The antihuman integrin $\alpha_{v} \beta_{3}$ antibody (clone LM609) used for immunofluorescence staining in this study cannot discriminate between low (inactive state)and high (activated state)-affinity conformations of integrin $\alpha_{v} \beta_{3}$ for RGD (26); therefore, human integrin $\alpha_{\mathrm{v}} \beta_{3}$ in the tumor xenografts may be inactivated (low affinity for ${ }^{99 \mathrm{~m}} \mathrm{Tc}-3 \mathrm{PRGD} 2$ ) after dasatinib treatment, though we did not observe these changes by immunofluorescence staining (Fig. 3C; Supplemental Fig. 3A).

We previously demonstrated that VEGF was a more sensitive biomarker than integrin $\alpha_{\mathrm{v}} \beta_{3}$ for antiangiogenic therapy monitoring (8), most likely because VEGF is released from tumor cells into the tumor microenvironment, and, therefore, tumor tissue penetration of the imaging probe is not essential (9). The VEGF NIRF probe (Dye755-Ran) used herein is human VEGF-specific and does not cross-react with murine VEGF (9). Therefore, the host vasculature status after Src inhibition was determined using 
the Matrigel plug assay and murine integrin $\beta 3$ staining. Dye755Ran could be simply adapted to produce radiolabeled probes for PET and SPECT by changing the NIRF dye to half-life-matched radionuclides such as ${ }^{89} \mathrm{Zr}$ and ${ }^{111} \mathrm{In}$, which can be used in the clinic. Indeed, VEGF-targeted PET imaging using ${ }^{89} \mathrm{Zr}$-labled antibodies has shown promise for tumor response monitoring in recent clinical trials (27-29).

By using molecular imaging approaches, we demonstrated the antiangiogenic effects and lack of cytotoxic effect of dasatinib. Logically, we hypothesized that, if used in combination with a cytotoxic agent such as DTX, dasatinib would show more effective antitumor efficacy in the dasatinib-resistant A549-fLuc mouse model. The in vivo therapy studies clearly verified the improved efficacy of combining antiangiogenic dasatinib therapy and cytotoxic DTX therapy; the antitumor efficacy of combination therapy was well-validated by ex vivo Ki-67 staining, and posttreatment imaging further confirmed that after combination therapy, the tumor signatures of glucose metabolic activity, integrin $\alpha_{v} \beta_{3}$ expression, and VEGF levels were all significantly reduced.

All the imaging agents used in this study, besides BLI, could be easily modified for clinical use. The NIRF probe could be adapted for PET and SPECT, and multiplexed imaging could then be performed in the same patient by carefully designing the imaging procedures. Alternatively, optical imaging could also be used for intraoperative imaging-guided tumor surgery. Taken together, the results of this study suggest that the combination of multiparameter imaging could provide a better understanding of simultaneous tumor signatures. A complete overview of all available tumor signatures would facilitate improved guidance for cancer therapy and may eventually lead to personalized cancer therapy being feasible.

\section{CONCLUSION}

We demonstrated the feasibility of using multiple molecular imaging approaches for target-specific and functional identification of multiple tumor signatures in vivo, which allowed the rational design of dasatinib combination tumor therapy. This approach could noninvasively determine tumor responses associated with cancer therapeutic interventions and may facilitate accelerating the development of new cancer therapies.

\section{DISCLOSURE}

The costs of publication of this article were defrayed in part by the payment of page charges. Therefore, and solely to indicate this fact, this article is hereby marked "advertisement" in accordance with 18 USC section 1734. This work was supported, in part, by "973" projects (2013CB733802 and 2011CB707705), National Natural Science Foundation of China (NSFC) projects (81471712, 81222019, 81125011, and 81371614), grants from Beijing Natural Science Foundation (7132131 and 7132123), and a grant from the Beijing Nova Program (Z121107002512010). No other potential conflict of interest relevant to this article was reported.

\section{REFERENCES}

1. Summy JM, Gallick GE. Src family kinases in tumor progression and metastasis. Cancer Metastasis Rev. 2003;22:337-358.

2. Parsons SJ, Parsons JT. Src family kinases, key regulators of signal transduction. Oncogene. 2004;23:7906-7909.
3. Araujo JC, Trudel GC, Paliwal P. Long-term use of dasatinib in patients with metastatic castration-resistant prostate cancer after receiving the combination of dasatinib and docetaxel. Cancer Manag Res. 2013;6:25-30.

4. Araujo JC, Trudel GC, Saad F, et al. Docetaxel and dasatinib or placebo in men with metastatic castration-resistant prostate cancer (READY): a randomised, double-blind phase 3 trial. Lancet Oncol. 2013;14:1307-1316.

5. Cai W, Chen X. Multimodality molecular imaging of tumor angiogenesis. J Nucl Med. 2008;49(suppl 2):113S-128S.

6. Rohren EM, Turkington TG, Coleman RE. Clinical applications of PET in oncology. Radiology. 2004;231:305-332.

7. Zhu Z, Miao W, Li Q, et al. ${ }^{99 \mathrm{~m} T c-3 P R G D 2}$ for integrin receptor imaging of lung cancer: a multicenter study. J Nucl Med. 2012;53:716-722.

8. Sun X, Ma T, Liu H, et al. Longitudinal monitoring of tumor antiangiogenic therapy with near-infrared fluorophore-labeled agents targeted to integrin alphavbeta3 and vascular endothelial growth factor. Eur J Nucl Med Mol Imaging. 2014;41:1428-1439.

9. Ma T, Liu H, Sun X, et al. Serial in vivo imaging using a fluorescence probe allows identification of tumor early response to cetuximab immunotherapy. Mol Pharm. 2015;12:10-17.

10. Liu Z, Sun X, Liu H, et al. Early assessment of tumor response to gefitinib treatment by noninvasive optical imaging of tumor vascular endothelial growth factor expression in animal models. J Nucl Med. 2014;55:818-823.

11. Cao Q, Cai W, Niu G, He L, Chen X. Multimodality imaging of IL-18-binding proteinFc therapy of experimental lung metastasis. Clin Cancer Res. 2008;14:6137-6145.

12. Liu Z, Liu H, Ma T, et al. Integrin $\alpha_{\mathrm{v}} \beta_{6}$-targeted SPECT imaging for pancreatic cancer detection. J Nucl Med. 2014;55:989-994.

13. Ma T, Sun X, Cui L, et al. Molecular imaging reveals trastuzumab-induced epidermal growth factor receptor downregulation in vivo. $J$ Nucl Med. 2014;55: 1002-1007.

14. Liu Z, Jia B, Shi J, et al. Tumor uptake of the RGD dimeric probe ${ }^{99 \mathrm{~m}} \mathrm{Tc}-\mathrm{G} 3-2 \mathrm{P} 4-$ RGD2 is correlated with integrin $\alpha_{\mathrm{v}} \beta_{3}$ expressed on both tumor cells and neovasculature. Bioconjug Chem. 2010;21:548-555.

15. Haubner R, Weber WA, Beer AJ, et al. Noninvasive visualization of the activated $\alpha_{\mathrm{v}} \beta_{3}$ integrin in cancer patients by positron emission tomography and [ ${ }^{18}$ F]galacto-RGD. PLoS Med. 2005;2:e70.

16. Dumont RA, Hildebrandt I, Su H, et al. Noninvasive imaging of $\alpha_{\mathrm{v}} \beta_{3}$ function as a predictor of the antimigratory and antiproliferative effects of dasatinib. Cancer Res. 2009;69:3173-3179.

17. Evans MJ. Measuring oncogenic signaling pathways in cancer with PET: an emerging paradigm from studies in castration-resistant prostate cancer. Cancer Discov. 2012;2:985-994.

18. Holland JP, Cumming P, Vasdev N. PET of signal transduction pathways in cancer. J Nucl Med. 2012;53:1333-1336.

19. Deroose CM, De A, Loening AM, et al. Multimodality imaging of tumor xenografts and metastases in mice with combined small-animal PET, small-animal CT, and bioluminescence imaging. J Nucl Med. 2007;48:295-303.

20. Smith-Jones PM, Solit D, Afroze F, Rosen N, Larson SM. Early tumor response to Hsp90 therapy using HER2 PET: comparison with ${ }^{18}$ F-FDG PET. J Nucl Med. 2006;47:793-796.

21. Sun X, Yan Y, Liu S, et al. ${ }^{18}$ F-FPPRGD2 and ${ }^{18}$ F-FDG PET of response to Abraxane therapy. J Nucl Med. 2011;52:140-146.

22. Kubota R, Yamada S, Kubota K, Ishiwata K, Tamahashi N, Ido T. Intratumoral distribution of fluorine-18-fluorodeoxyglucose in vivo: high accumulation in macrophages and granulation tissues studied by microautoradiography. $\mathrm{J} \mathrm{Nucl}$ Med. 1992;33:1972-1980.

23. Balkwill F, Mantovani A. Inflammation and cancer: back to Virchow? Lancet. 2001;357:539-545.

24. Schade AE, Schieven GL, Townsend R, et al. Dasatinib, a small-molecule protein tyrosine kinase inhibitor, inhibits T-cell activation and proliferation. Blood. 2008;111:1366-1377.

25. Klinghoffer RA, Sachsenmaier C, Cooper JA, Soriano P. Src family kinases are required for integrin but not PDGFR signal transduction. EMBO J. 1999;18: 2459-2471.

26. Kiosses WB, Shattil SJ, Pampori N, Schwartz MA. Rac recruits high-affinity integrin $\alpha_{v} \beta_{3}$ to lamellipodia in endothelial cell migration. Nat Cell Biol. 2001;3:316-320.

27. Gaykema SB, Schroder CP, Vitfell-Rasmussen J, et al. ${ }^{89} \mathrm{Zr}$-trastuzumab and ${ }^{89} \mathrm{Zr}$-bevacizumab PET to evaluate the effect of the HSP90 inhibitor NVP-AUY922 in metastatic breast cancer patients. Clin Cancer Res. 2014;20:3945-3954.

28. Oosting SF, Brouwers AH, van Es SC, et al. ${ }^{89} \mathrm{Zr}$-bevacizumab PET visualizes heterogeneous tracer accumulation in tumor lesions of renal cell carcinoma patients and differential effects of anti-angiogenic treatment. J Nucl Med. 2015;56:63-69.

29. van Asselt SJ, Oosting SF, Brouwers AH, et al. Everolimus reduces ${ }^{89} \mathrm{Zr}$-bevacizumab tumor uptake in patients with neuroendocrine tumors. J Nucl Med. 2014;55: 1087-1092. 